\title{
Effectiveness of rehabilitation
}

\section{interventions for people with multiple sclerosis - A Cochrane Review summary with commentary}

\author{
Bhasker Amatya ${ }^{\mathrm{a}, \mathrm{b}, *}$, Fary Khan ${ }^{\mathrm{a}, \mathrm{b}}$ and Mary Galea ${ }^{\mathrm{a}, \mathrm{b}}$ \\ a Department of Medicine (Royal Melbourne Hospital), The University of Melbourne \\ ${ }^{\mathrm{b}}$ Department of Rehabilitation and Australian rehabilitation Research Centre, Royal Melbourne Hospital, \\ Parkville, VIC, Australia
}

\begin{abstract}
The aim of this commentary is to discuss the rehabilitation perspective in the recently published Cochrane Review "Rehabilitation for people with multiple sclerosis: an overview of Cochrane Reviews" by Amatya, Khan \& Galea ${ }^{1}$, under the direct supervision of Multiple Sclerosis and Rare Diseases of the CNS group. This Cochrane Corner is produced in agreement with "NeuroRehabilitation" by Cochrane Rehabilitation.
\end{abstract}

Keywords: Multiple sclerosis, rehabilitation, disability, impairment

\section{Background}

Multiple sclerosis (MS) is a major cause of chronic neurological disability in young and middle-aged adults (aged 18 to 50 years), with a significant progressive long-term disability burden (Beer, Khan, \& Kesselring, 2012). People with MS (pwMS) can

\footnotetext{
*Address for correspondence: Bhasker Amatya, E-mail: bhasker.amatya@mh.org.au.

${ }^{1}$ The abstract/plain language summary of this Cochrane Review is taken from a Cochrane Review previously published in the Cochrane Database of Systematic Reviews 2019, Issue 1, URL: http://dx.doi.org/10.1002/14651858.CD012732 (see www.cochranelibrary.com for information). Cochrane Reviews are regularly updated as new evidence emerges and in response to feedback, and Cochrane Database of Systematic Reviews should be consulted for the most recent version of the review.
}

present with various combinations of functional and cognitive deficits, and other issues such as fatigue, pain, and incontinence, which are amenable to comprehensive rehabilitation (Bennett, 2010). Many rehabilitation interventions have been trialed in pwMS (Amatya, La Mantia, Demetrios, \& Khan, 2013).

Rehabilitation for people with multiple sclerosis: an overview of Cochrane Reviews

(Amatya, Khan \& Galea, 2019)

\section{Objective}

This review evaluated evidence from published Cochrane Reviews of clinical trials to summarise the evidence regarding the effectiveness and safety 
of rehabilitation interventions for pwMS, to improve patient outcomes.

\section{What was studied and methods}

A comprehensive search of the Cochrane Database of Systematic Reviews (up to December 2017) was performed to identify Cochrane Reviews that assessed the effectiveness of organised rehabilitation interventions for pwMS. Two reviewers independently assessed the quality of included reviews, using the Revised Assessment of Multiple Systematic Reviews (R-AMSTAR) tool, and the quality of the evidence for reported outcomes, using the Grades of Recommendation, Assessment, Development and Evaluation (GRADE) tool.

\section{Results}

Fifteen Cochrane systematic reviews comprising 164 randomised controlled trials (RCTs) and four controlled clinical trials (CCTs), with a total of 10,396 participants were included. The included reviews evaluated a wide range of rehabilitation interventions, including: physical activity and exercise therapy, hyperbaric oxygen therapy (HBOT), wholebody vibration, occupational therapy, cognitive and psychological interventions, nutritional and dietary supplements, vocational rehabilitation, information provision, telerehabilitation, and interventions for the management of spasticity. All reviews were assessed to be of high to moderate methodological quality, based on R-AMSTAR criteria.

The qualitative synthesis of 'best evidence' shows moderate-quality evidence for physical therapeutic modalities (exercise and physical activities) in improving functional outcomes (mobility, muscular strength, aerobic capacity), reducing impairment (fatigue), and improving participation (quality of life). There was moderate-quality evidence for inpatient or outpatient multidisciplinary rehabilitation programs leading to longer-term gains at the levels of activity and participation, and for interventions providing information about MS and its management in improving patient knowledge. There was low-quality evidence for neuropsychological interventions, symptom-management programs (spasticity), whole body vibration, telerehabilitation and vocational intervention in improving some patient outcomes. Evidence for other rehabilitation modalities was inconclusive, due to lack of robust studies.

\section{Conclusions}

Despite the broad range of rehabilitative treatments available to treat pwMS, there was a lack of high-quality evidence showing the effectiveness of various modalities. The available evidence suggests that structured, multidisciplinary rehabilitation programs and physical therapy (exercise or physical activities) can improve functional outcomes (mobility, muscle strength, aerobic capacity), and quality of life. More studies with robust methodology are still needed to justify the use of many other interventions. These findings, however, should be interpret cautiously, as most of the included reviews could be considered out of date or are yet to be updated, implying that recent trials may have been missed.

\section{Implications for practice in neurorehabilitation}

People with MS have complex care needs, due to cumulative effects of impairments and disabilities, requiring comprehensive disease management, including rehabilitation. A rehabilitation approach to pwMS may include a wide range of treatments and interventions. Evidence presented in this overview of Cochrane Reviews supports the strategy that suggests varied approaches to rehabilitation. Exercise or physical activities probably improve patients' functions such as mobility, muscle strength, aerobic capacity and quality of life and probably reduce patient-reported fatigue. A comprehensive multidisciplinary rehabilitation approach showed longer-term gains in activity and participation. Provision of MS-related information to the patients helped in increasing patients' knowledge. Neuropsychological interventions, symptom-management programs for spasticity, whole body vibration and telerehabilitation may slightly improve some patient outcomes. The evaluated rehabilitation interventions were generally considered to be safe; however, many studies did not report adverse effects. This suggests that regular specialist evaluation and follow-up to assess the needs of patients with all types of MS for appropriate rehabilitation interventions may be of benefit, although the certainty 
of evidence varies across the different types of interventions.

\section{Acknowledgments}

The author are grateful to the Cochrane Multiple Sclerosis and Rare Diseases Group for their support and assistance and would like to thank Cochrane Rehabilitation and NeuroRehabilitation for reviewing the contents of the Cochrane Corner.

\section{Conflict of interest}

The author declares no conflicts of interest.

\section{References}

Amatya, B., Khan, F., \& Galea, M. (2019). Rehabilitation for people with multiple sclerosis: An overview of Cochrane Reviews. Cochrane Database of Systematic Reviews 2019(1). Art. No.: CD012732. URL: http://dx.doi.org/10.1002/146518 58.CD012732

Amatya, B., La Mantia, L., Demetrios, M., \& Khan, F. (2013). Non pharmacological interventions for spasticity in multiple sclerosis. Cochrane Database of Systematic Reviews, (2). Art. No.: CD009974. DOI: 10.1002/14651858.CD009974.pub2.

Beer, S., Khan, F., \& Kesselring, J. (2012) Rehabilitation interventions in multiple sclerosis: An overview. Journal of Neurology, 259, 1994-2008.

Bennett, S.E. (2010) Effective rehabilitation methods in patients with multiple sclerosis. US Neurology, 5(2), 67-70. 